\title{
Discussion on IOT Business Mode Based on Cloud Computing
}

\author{
Fangning Chen, Yizeng Chen
}

\author{
School of Management, Shanghai University, Shanghai, 200444, China
}

Keywords: Cloud computing, Internet of Things, Industrial chain, Business mode, Structure

\begin{abstract}
With technological development, Internet of Things (IOT) adds intelligent characteristic. It integrates multiple novel properties, such as positioning recognition, tracking inspection and control and novel product adoption. The industrial chain constructed by IOT is gradually popularized and cannot break away from the support of cloud computing. Cloud computing owns new prominent advantages such as calculation process advantage. Cloud computing can be integrated in wider IOT and more appropriate business mode can be explored. Under the promotion of operators, IOT creates a new mode, which coincides with business trend in the new stage. IOT sets up an essential platform to share resources available in the internet and enhance competition strength from the overall architecture.
\end{abstract}

\section{Introduction}

IOT is related to multiple industries under the emerging state and involves multiple processes in daily life. Business follows industrial chain, and IOT covers more fields in the market structure. Industrial train brings a new industry under cluster situation. However, IOT in progress still has defects and loopholes which hinder long-term business progress ${ }^{[1]}$. To boost lasting business progress and optimize existing industrial chain, a better new business mode is required. If mode configuration is reset, multiple parties can gain win-win and income and increase the return in industrial chain.

\section{Analysis of IOT industrial chain}

\section{Features of IOT}

IOT contains the following connotations: recognize radio frequency and scan, precisely position globally, scan with the help of laser, draft protocol and induce with the help of infrared ray. Goods are included in IOT where they may share information smoothly. IOT recognizes things according to intelligent approach and sets precise positioning. IOT integrates monitoring in each time bucket and internet regulation on the basis of tracking. The emerging technology covers IOT which belongs to essential technology. IOT is closely connected with existing network and an entire whole is constructed. Based on this, more extensive follow-up networks can be gained by expanding IOT, which follows industrial chain gained by IOT extension. IOT is convenient for sharing and transferring information. The client is integrated in the network.

\section{Features of Industrial Chain}

IOT includes perception layer, middle transmission layer and essential processing layer. The three layers are integrated into a whole and cover all information. The system gained from IOT integration is related to daily life and production, and the industrial chain is huge. The perception layer acquires initial induction information. Similar to the five sense organs, it identifies the things which can be gained by induction and can summarize information. The middle layer accepts access accessories in information and deployment network, and it further approximates central nervous system. Relatively, the application layer connects the above two layers and owns prominent specialty tendency. It lays particular emphasis on differentiating and analyzing information and then drafting decisions. From a wider perspective, new network under intelligent path supplies essential reference for decision making.

IOT covers industrial chain under complex architecture and contains subdivided multiple processes. However in China, IOT is still in initial progress and its expansion is supported by operators. Compared with developed countries, the industrial chain attached to IOT has many 
deficient and blank sections, and middle process available is deficient. IOT market extends initial overall scale, but it still remains expanding ${ }^{[2]}$. (III) Existing Multi-link of Industrial China

Firstly, chain-supported supply object. Suppliers provide induction recognition accessories and a complete set of intra-internet equipment. IOT cannot lack such induction accessories and brings convenience of identifying information and collecting essential. Among them, induction accessories contain the sensor, recognition accessories peculiar to RFID and matched devices with intelligent characteristic. The end of the perception layer is also equipped with typical accessories such as RFID for radio frequency identification in routine process, execution device, equipment selected for intelligent regulation, positioning under GPS architecture and products within the network.

Secondly, network and software in the network. IOT can transfer numerical value and supply fundamental support. It covers telecommunication network, conventional network, dedicated network and power grid within the section. The perception layer can lay microoperation-peculiar system, preliminarily gather the information and process information. Besides, it can supply middleware. The numerical values gathered fall into the induction layer. In this way, intra-network information which is uniformly deployed can be gained through collection, and such information is interconnected and shared in real time. Moreover, it drafts accurate semantics available for operation, eliminates diversified obstacles in interaction process and enhances convenience for follow-up processing. Additionally, solution scheme available is set. Such scheme can be applied in diversified fields. As well, it supplies solutions.

Thirdly, operation of equipped services in each step. According to customer demand, soft and hardware in IOT architecture are integrated into a whole. The novel scheme set by the manufacturer furthers soft hardware users. Aiming at operation service, it contains network connection in each time bucket, new platform for layout, authentication and testing, daily service for consultation supply and charging service. It can be regulated and accessed in the terminal. Intra-network service integrates conventional service under operation situation, industrial essential application and other related links.

\section{Cloud Computing in New Time Bucket}

\section{Connotation of Cloud Computing}

Network application cannot be separated from cloud computing, and it belongs to application mode. In a narrow sense, cloud computing is based on the laid infrastructure and includes delivery in IT process and follow-up application. Cloud computing accords with the demand, and essential integrated recourses can be gained through expansion. Correspondingly, cloud computing covers delivery steps and supported follow-up application in a broad sense. Cloud computing can be easily expanded and also conforms to deep-layer needs. Network integrates all resources which can be deployed and deploy them uniformly. In this way, wider resource pool is created to accept corresponding service according to the demand ${ }^{[3]}$.

IOT is faced with extensive existing objects and also own more potential users. In collaborative management, each department cooperates with each other closely and takes charge of dispatching and commanding in daily situation. IOT integrates multiple enterprises and contains more potential users. In accordance with detailed goal, marketing can be divided into diversified categories. In current state, IOT creates novel business mode, such as platform operation, industrial alliance, routine service of application type. Existing mode tends to be monotonous and has prominent defects. It is limited to narrow business perspective, and fails to accept logic route to main network and to add new value. For value analysis, usable tools are deficient. The existing mode often ignores deep-layer interactions and only follows operation perspective. For integrators and equipment supply in the market, there is short of deepened excavation.

\section{Unique Advantage Value of Cloud Computing}

\section{Value of Cloud Aggregation}

As a new concept, cloud aggregation is rooted in current cloud computing. It sets user center, extends the scope of business with the help of the operation platform laid, integrates target market and re-integrates all internal and external resources. The business mode creates the common new value, involving users, relevant merchants ad other participant subjects. Cloud computing is integrated in 
network architecture, which boosts calculation speed and supplies the foundation for long-term development of essentials. Fundamentally, cloud aggregation evades the prominent obstacles of network and overall adopts cloud computing. Cloud computing is making progress rapidly, and the two are correlated.

Cloud aggregation adds dynamic and real-time intra-network regulation, dynamically controls existing diversified things in the network and sets intelligent process analysis. The novel mode is rooted in cloud aggregation which adds new intelligent elements for existing environment and adopts new technological approach of IOT.

Route Achieved by Exploration

Cloud computing integrates existing cloud clusters and covers operators, cloud computing objects, supply subjects of essentials, integrators, and subjects that market investment follows. The functions within the normal route should be valued, and new cycle of benign situation is created. Sensing accessories construct more extensive network foundation. The sensor can be divided into microprocessor, tiny unit of perceiving essentials and chips available for communication. The sensor can check conventional process and consume low gross power dissipation. Cloud computing sets up cloud platform in the new time bucket, and the storage process is set to distributed storage. The existing nodes in network are called, and the information gathered is convenient for subsequent calculation. IOT brings convenience for normal operation, interconnects and shares information with the help of virtualization ${ }^{[4]}$.

Long-term Market Advantage

Cloud computing creates a new mode which can be adopted by business and adds unique value of IOT. In such state, value network integrates the value chain peculiar to each link. The value chain interconnects linear, upstream and downstream information, spans the boundary that enterprises present, and adds customer group and market impetus. IOT closely connects existing things within the framework of industrial chain, regulates dynamic information in real time and precisely delivers such information. The information can be summarized, and the conclusion gained is used for treatment. The objects in the multiple links can share information, and internal communication is smoother. This brings convenience for gaining useful information and adding more gather market value.

\section{Defects of Existing Mode}

IOT brings convenience for smooth communication in the industry. The services set by IOT are laid in multiple links. In such mode, users may screen a service they like. Seeing from existing state, IOT owns favorable overall environment and extensive prospect. However, the industry lacks competitive strength, and core level is still low. The new system fails to be improved, and there is short of industrial chain under integration state. In detail, industrial chain connects multiple industries and fails to closely cooperate. Thus, high total sum is consumed. Besides, internal chain and corresponding external chain fail to be matched. It is very difficult for the industry to really acquire the expected income, and potential environmental threat fails to clearly specified.

Diversified modes of IOT can be divided into the following categories: the first kind of approaches is that the channel is laid for telecom operation so that users contact network through the set channel; the second kind of approaches is promotion under development, and merchants cooperate with each other to create modes. In such mode, telecom merchants set up the platform and take charge of deploying platform operation and collecting expenditure. The third kind of approaches is to bring convenience for operation with the help of platform. The equipment is connected to existing platform, and the interface is open. It is faced with application service subjects. In addition, it can supply public essential service, and adds industrial alliance. After a strong enterprise constructs such alliance, it can integrate all resources in the chain and span multiple fields.

In existing network architecture, operators set up interactive direct route which contains diversified protocol services. The collaboration procedure peculiar to integrators can be supplied, and convenient and fast channel can be set up. The existing modes can be divided into: service channel of directly supply, connection of integrators and operation subjects, application industry alliance and 
network service in the platform. Radically, they lack creation, purely follow technological perspective, fail to consider normal detail restoration, also lack industrial logic which can be checked, ignore equipment manufacturers and relevant integrators and lack close customer interaction. In such situation, industrial chain gradually shows unbalance tendency.

\section{Exploration of Most Appropriate New Business Structure}

IOT owns the following unique attributes: it reconstructs information, aggregates such information, supplies aggregation service within normal steps and reconstructs all value. Compared with the old mode, IOT-related industrial chain accepts suppliers. The leaders in the chain should be deemed as such suppliers. IOT gradually tends towards cloud platform, optimizes and resets complete industrial chain and depends on reconstructed platform. If users fail to own independent technology, such platform constructed by IOT becomes effective. This is because when conditions are permitted, operation platform follows cloud operation, and the industries in IOT architecture are integrated.

The construction of industrial chain should strive for being more complete. It contains such equipment as full sets of devices, software and hardware. Industrial chain starts from products and contains services and information which can be transferred. In each level, IOT is equipped with necessary hardware and relevant software. The precise scheme peculiar to systematism is drafted, and the network includes operators, equipment suppliers, system integrators and merchants supplying operation service. Operators depend on well-constructed cloud platform to integrate diversified resources. By the processed process, numerical values peculiar to cloud services are kept in the data pool. In accordance with customer tendency, new terminal that network adds supplies precise information which is gained by screening. In such platform, all merchants should be able to interact smoothly, which avoids information transfer under asymmetry situation and expands the information which can be shared ${ }^{[5]}$.

IOT extension can gain higher-level new mode. In detail, it integrates multi-source information, extends management radius, sets real-time regulation and lays particular emphasis on supplying diversified service. In this way, the chain mode in the single step is discarded, and new net-shaped mode is created. After diversified industries are optimized, the network owns more prominent permeability characteristics and penetrates in more fields. Meanwhile, more additional industrial value is added.

\section{Conclusions}

In the information age, IOT integrates the information transmitted by multiple industries and extends the coverage. But seeing from current situation, IOT still lacks scale and fails to seek out the optimal mode. IOT based on cloud computing tallies with industrial chain, and IOT expansion drives the industrial chain under extension and integrates detailed multiple branches. Besides, the novel mode under win-win architecture is created, and business process is integrated. Thus, appropriate business structure is gained.

\section{References}

[1] Huang Yi, Deng Shaoling, Cloud Aggregation - Innovation of IOT Business Mode. Information System Engineering, 2012 (02): 132-134.

[2] Hu Baoliang, Study on Components of IOT Business Mode Based on Canvas Model. Technological Economy, 2015 (02):44-49.

[3] Wang Kai, Fan Pengfei, Huang Weidong, Study on business mode where telecom operators develop IOT under perspective of industrial chain. Journal of Chongqing University of Posts and Telecommunications (Social Sciences), 2013 (01):96-101. 
[4] Yin Liying, Wei Ming, Construction of IOT Business Mode of Telecom Operators - Based on Oswald Framework. China Business and Market, 2013 (06):92-96.

[5] Xie Qin, Analysis of IOT Industrial Chain Business Mode Structure Based on Cloud Computing. Guide to Business, 2013 (11):170. 